\title{
National perioperative outcomes of pulmonary lobectomy for cancer in the obese patient: A propensity score matched analysis
}

\author{
Hunter Launer, BA, ${ }^{\mathrm{a}}$ Danh V. Nguyen, $\mathrm{PhD},{ }^{\mathrm{b}}$ and David T. Cooke, $\mathrm{MD}^{\mathrm{a}}$
}

Objectives: Obesity in the United States is a growing epidemic that results in challenging patients with complicated comorbidities. We sought to compare hospital outcomes of obese patients with those of nonobese patients undergoing pulmonary lobectomy for cancer.

\begin{abstract}
Methods: We performed a retrospective cohort analysis of obese (body mass index $\geq 30 \mathrm{~kg} / \mathrm{m}^{2}$ ) and nonobese (body mass index $<30 \mathrm{~kg} / \mathrm{m}^{2}$ ) patients undergoing pulmonary lobectomy for lung cancer. By using the Nationwide Inpatient Sample database from 2002 to 2007, we determined independent risk factors for perioperative death, discharge to an institutional care facility, and prolonged hospital length of stay ( $>14$ days). Cohorts were matched on the basis of propensity scores incorporating preoperative patient variables.
\end{abstract}

Results: We identified 1238 obese patients (3.7\%) and 31,983 nonobese patients $(96.3 \%)$ undergoing lobectomy for cancer. In regard to patient demographics, obese patients were younger (mean age, 64.8 vs 66.7, $P<.001)$ and predominantly female $(59.5 \%$ vs $50.0 \%, P<.001)$ compared with nonobese patients. After matching based on propensity scores, except for a greater incidence of pulmonary insufficiency $(P=.03)$ and pneumonia $(P=.01)$ in the obese group, there were no differences in postoperative complications. By controlling for patient demographics, obese patients had higher odds to be discharged to an institutional care facility (odds ratio, 1.21; $P=.02$ ) but not for prolonged hospital length of stay or perioperative death.

Conclusions: Obese patients have an increased risk for postoperative pulmonary complications but not other morbidity, mortality, or prolonged hospital length of stay after lobectomy for cancer. Obesity should not be considered a surgical risk factor for pulmonary resection. (J Thorac Cardiovasc Surg 2013;145:1312-8)

Earn CME credits at

http://cme.ctsnetjournals.org

Obesity in the United States has increased from an incidence of $15 \%$ in 1980 to more than $35 \%$ in 2010 , with more than $65 \%$ of US residents currently overweight or obese. ${ }^{1,2}$ Obesity may pose risks to surgery that are not present in the nonobese population. Medical conditions associated with obesity, such as hypoventilation syndromes, cardiac arrhythmias, wound healing complications, and proinflammatory states, cause clinicians to be hesitant to proceed with surgery as a means of complex cancer treatment. ${ }^{3,4}$ Previous studies report an

\footnotetext{
From the Division of Cardiothoracic Surgery, ${ }^{\text {a }}$ Department of Surgery, University of California, Davis Medical Center, and Division of Biostatistics, ${ }^{\mathrm{b}}$ Department of Public Heath Sciences, University of California, Davis, Sacramento, Calif.

This publication was made possible by the National Center for Advancing Translational Sciences, National Institutes of Health, through Grant UL1 TR000002. Its contents are solely the responsibility of the authors and do not necessarily represent the official view of the National Center for Advancing Translational Sciences or National Institutes of Health.

Disclosures: Authors have nothing to disclose with regard to commercial support.

Received for publication April 3, 2012; revisions received Sept 18, 2012; accepted for publication Oct 2, 2012; available ahead of print Nov 12, 2012.

Address for reprints: David T. Cooke, MD, Division of Cardiothoracic Surgery, University of California, Davis Medical Center, 2221 Stockton Blvd, Suite 2117, Sacramento, CA 95817 (E-mail: david.cooke@ucdavis.ucdmc.edu).

0022-5223/\$36.00

Copyright (c) 2013 by The American Association for Thoracic Surgery

http://dx.doi.org/10.1016/j.jtcvs.2012.10.012
}

increase in postoperative morbidity in the obese patient after nonbariatric surgery, although not perioperative or 30-day mortality. ${ }^{5,6}$

The impact of obesity on general thoracic surgery outcomes is unclear. Single institution studies suggest that obesity may not be a risk factor for pulmonary lobectomy and esophagectomy for postoperative complications and mortality. ${ }^{7,8}$ We queried the Healthcare Cost and Utilization Project Nationwide Inpatient Sample (HCUP-NIS) database and used a propensity score (PS) matched analysis to compare perioperative mortality and morbidity rates between nonobese and obese patients undergoing pulmonary lobectomy for lung cancer. In addition, we compared the incidence of discharge to an institutional care facility (ICF) and rate of prolonged length of stay (PLOS).

\section{MATERIALS AND METHODS Database}

This is a retrospective cohort analysis of hospital discharge data for the years 2002 to 2007, from the HCUP-NIS. The HCUP-NIS is the largest all-payer inpatient care database in the United States. HCUP-NIS is an approximate $20 \%$ stratification of US hospitals representing 44 states, 1050 hospitals, and approximately 8 million hospital stays. ${ }^{9}$ This project was approved by the institutional review board of the University of California, Davis Medical Center.

\section{Patient Selection and Search Criteria}

Patients were identified by procedure codes, and preoperative and postoperative morbidity were identified by disease codes. All procedure and 


\begin{tabular}{|c|c|}
\hline \multicolumn{2}{|c|}{ Abbreviations and Acronyms } \\
\hline BMI & $=$ body mass index \\
\hline CHF & $=$ congestive heart failure \\
\hline $\mathrm{CI}$ & $=$ confidence interval \\
\hline HCUP & $\begin{aligned} \mathrm{S}= & \text { Healthcare Cost and Utilization } \\
& \text { Project Nationwide Inpatient Sample }\end{aligned}$ \\
\hline ICD-9 & $\begin{aligned} I= & \text { International Classification of } \\
& \text { Diseases, 9th edition, Clinical } \\
& \text { Modification }\end{aligned}$ \\
\hline ICF & $=$ institutional care facility \\
\hline OR & $=$ odds ratio \\
\hline PLOS & $=$ prolonged length of stay \\
\hline PS & $=$ propensity score \\
\hline
\end{tabular}

disease codes were based on the International Classification of Diseases, 9th edition, Clinical Modification (ICD-9-CM) Volume 3. The overall study population included patients who received pulmonary lobectomy (ICD-9-CM codes 32.40, 32.41, and 32.49) for the primary diagnosis of lung cancer (ICD-9-CM codes 162.0-162.9, 197.0-197.3). From this population, patients were divided into 2 cohorts on the basis of body mass index (BMI): nonobese $\left(\mathrm{BMI}<30 \mathrm{~kg} / \mathrm{m}^{2}\right)$ and obese $\left(\mathrm{BMI} \geq 30 \mathrm{~kg} / \mathrm{m}^{2}\right)$.

\section{Patient and Hospital Demographics and Perioperative Outcomes}

Patient demographics studied included age, sex, race, primary mode of payment, and preoperative comorbidities. Preoperative comorbidities measured were diabetes mellitus, hypertension, coronary artery disease, congestive heart failure (CHF), peripheral vascular disease, chronic obstructive pulmonary disease, gastroesophageal esophageal reflux disease, and chronic kidney disease. The hospital demographic measured was hospital type (ie, rural, urban nonteaching and urban teaching). Perioperative outcomes measured included occurrence of PLOS as defined by hospital stay more than 14 days, discharge disposition (routine to home, ICF, or death at the time of discharge), and postoperative morbidity. Postoperative morbidity included cardiac complications, respiratory complications, nervous system complications, renal complications, gastrointestinal complications, vascular complications, surgical wound complications, and other. A list of ICD-9-CM codes for all measured pre- and postoperative comorbidities is found in Appendix 1. For the majority of the preoperative comorbidity ICD-9-CM codes analyzed, their listing for a patient discharge is most likely preprocedure; thus, it is impossible to know definitively on the basis of the deidentified design of the NIS database.

\section{Statistical Analysis}

Differences in the distribution of patient disposition/outcome (routine [to home], discharged to ICF, or died), patient demographics (including age, sex, race, primary expected payer, and hospital type), and pre- and postoperative comorbidities were evaluated using the $t$ test for continuous variables and chi-square test for proportions. The primary analyses examine the association between obesity status and likelihood of discharge to an ICF, mortality, and PLOS. For this purpose, the PS method was used to obtain matched sets of nonobese patients to each obese patient on the basis of PS matching. For this study, the PS is the probability of being obese as a function of preoperative patient variables, and the estimated PS was obtained using logistic regression. ${ }^{10,11}$ The PS-matched sets were then used in logistic regression models to evaluate the likelihood (odds) of discharge to ICF (relative to routine). Likewise, logistic regression was used to evaluate the odds of dying. For PLOS, which potentially depends on patient disposition/outcome (routine, discharged to ICF, or died), logistic regression models were considered for data stratified by patient disposition. All analyses were performed using SAS 9.2 (SAS Institute Inc, Cary, NC). All $P$ values reported are for 2 -sided tests.

\section{RESULTS \\ Patient Demographics}

The study cohorts consisted of 1238 obese patients $(3.7 \%)$ and 31,983 nonobese patients $(96.3 \%)$ (Table 1$)$. From 2002 to 2007 , there was a $32.7 \%$ increase in the number of obese patients undergoing lobectomy for lung cancer, with obese patients accounting for $2.8 \%$ of total lobectomies in 2002 to $4.6 \%$ in 2007. Obese and nonobese patients both demonstrated a similar mean age (64.8 vs 66.7 years) and predominantly white race $(85.0 \%$ vs $86.5 \%)$. A higher percentage of obese patients were female than nonobese patients $(59.5 \%$ vs $50.0 \%, P<.001)$. The majority of all patients had Medicare/Medicaid as the primary expected payer, with nonobese patients exhibiting a higher predilection toward Medicare/Medicaid compared with obese patients $(62.9 \%$ vs $58.8 \%, P=.006)$. A higher number of nonobese patients had their surgery performed at urban teaching hospitals $(55.2 \%$ vs $50.3 \%, P=.002)$. Obese patients had more preoperative comorbidities (Table 1). Obese patients had higher rates of diabetes mellitus, hypertension, coronary artery disease, CHF, and gastroesophageal esophageal reflux disease (all $P \leq .003$ ).

\section{Perioperative Outcomes}

On the basis of the cohort of PS-matched sets, there were more obese patients $(31.0 \%)$ than nonobese patients $(26.7 \%)$ discharged to an ICF and higher perioperative mortality in the nonobese group compared with the obese cohort ( $2 \%$ vs $1.2 \%$; Table 2 ). PLOS was more common among nonobese patients $(8.6 \%)$ relative to obese patients $(6.4 \%$; $P=.01)$. There was no difference in postoperative morbidity among obese and nonobese patients except for pulmonary insufficiency and pneumonia, which were more prevalent in the obese cohort $(9.5 \%$ vs $7.7 \%, P=.03$ and $19.7 \%$ vs $16.7 \%, P=.01$, respectively; Table 2 ).

\section{Odds of Discharge to an Institutional Care Facility, Perioperative Mortality, and Prolonged Length of Stay}

On the basis of PS-matched sets, we examined the likelihood (odds) of patients being discharged to an ICF relative to routine discharge to home among obese and nonobese patients, adjusted for patient demographics (including age, sex, race, primary expected payer, and hospital type) and pre- and postoperative morbidities (Table 3 ). Obese patients were more likely to be discharged to an ICF odds ratio [OR], 1.21 (95\% confidence interval [CI], 1.03-1.43; $P=.02)$. Increasing age, female sex, and black race were associated with increased odds of discharge to an ICF. The odds of 
TABLE 1. Patient demographics

\begin{tabular}{|c|c|c|c|c|c|c|}
\hline \multirow[b]{2}{*}{ Variable } & \multicolumn{3}{|c|}{$\begin{array}{c}\text { Obese } \\
(\mathbf{n}=\mathbf{1 2 3 8})\end{array}$} & \multicolumn{2}{|c|}{$\begin{array}{c}\begin{array}{c}\text { Nonobese } \\
(\mathrm{n}=\mathbf{3 1}, \mathbf{9 8 3})\end{array} \\
\end{array}$} & \multirow{2}{*}{$\begin{array}{c}P \\
\text { value }\end{array}$} \\
\hline & Cou & & $\%$ & Count & $\%$ & \\
\hline Age, mean (SD) & 64.8( & & & $66.7(10.5$ & & $<.0$ \\
\hline \multicolumn{7}{|l|}{ Sex } \\
\hline Male & 501 & & 40.5 & 15,965 & 50.0 & $<.0$ \\
\hline Female & 737 & & 59.5 & 15,987 & 50.0 & \\
\hline \multicolumn{7}{|l|}{ Race } \\
\hline White & 801 & & 85.0 & 20,604 & 86.5 & .1 \\
\hline Black & 76 & & 8.1 & 1528 & 6.4 & \\
\hline Other & 65 & & 6.9 & 1698 & 7.1 & \\
\hline \multicolumn{7}{|l|}{ Type of hospital } \\
\hline Rural & 60 & & 4.8 & 1847 & 5.8 & .0 \\
\hline Urban nonteaching & 555 & & 44.8 & 12,478 & 39.0 & \\
\hline Urban teaching & 623 & & 50.3 & 17,658 & 55.2 & \\
\hline \multicolumn{7}{|l|}{$\begin{array}{l}\text { Primary expected } \\
\text { payer }\end{array}$} \\
\hline Medicare/Medicaid & 727 & & 58.8 & 20,101 & 62.9 & .0 \\
\hline Private/HMO & 467 & & 37.8 & 10,714 & 33.5 & \\
\hline Self & 23 & & 1.9 & 463 & 1.4 & \\
\hline Other & 20 & & 1.6 & 677 & 2.1 & \\
\hline \multirow{2}{*}{$\begin{array}{c}\text { Preoperative } \\
\text { morbidity } \\
\end{array}$} & \multicolumn{3}{|c|}{$\begin{array}{c}\text { Obese } \\
(\mathbf{n}=\mathbf{1 2 3 8})\end{array}$} & \multicolumn{2}{|c|}{$\begin{array}{c}\text { Nonobese } \\
(\mathrm{n}=\mathbf{3 1}, 983)\end{array}$} & 1 \\
\hline & Count & $\%$ & & Count & $\%$ & val \\
\hline Diabetes mellitus & 31 & 2.5 & & 233 & 0.7 & $<.00$ \\
\hline Hypertension & 809 & 65.3 & & 14,305 & 44.7 & $<.00$ \\
\hline $\begin{array}{l}\text { Coronary artery } \\
\text { disease }\end{array}$ & 243 & 19.6 & & 5084 & 15.9 & \\
\hline $\mathrm{CHF}$ & 76 & 6.1 & & 1388 & 4.3 & .0 \\
\hline PVD unspecified & 34 & 2.7 & & 1161 & 3.6 & .1 \\
\hline COPD & 488 & 39.4 & & 12,261 & 38.3 & .44 \\
\hline GERD & 223 & 18.0 & & 4080 & 12.8 & $<.00$ \\
\hline $\begin{array}{l}\text { Chronic kidney } \\
\text { disease }\end{array}$ & 16 & 1.3 & & 311 & 1.0 & .2 \\
\hline
\end{tabular}

$\overline{S D \text {, Standard deviation; } H M O \text {, Health Maintenance Organization; } C H F \text {, congestive }}$ heart failure; $P V D$, peripheral vascular disease; $C O P D$, chronic obstructive pulmonary disease; GERD, gastroesophageal reflux disease.

discharge to an ICF for patients with private/Health Maintenance Organization and self-pay primary expected payer type were lower compared with Medicare/Medicaid. Except for pneumonia and gastrointestinal complications, all other postoperative morbidities were significantly associated with increased odds of discharge to an ICF.

Likewise, on the basis of PS-matched data, we assessed the likelihood of perioperative death among obese and nonobese patients, adjusted for patient demographics and pre- and postoperative morbidities (Table 4). Obesity status was not associated with perioperative death (OR, 0.63; 95\% CI, 0.33-1.18; $P=.15$ ). Other factors predictive of reduced likelihood of perioperative mortality include decreasing age and urban hospital (both teaching and nonteaching) relative to rural hospital (all $P \leq .03$ ). Preoperative CHF was associated with increased odds of death (OR, 1.96; 95\% CI, 1.06-3.61;
TABLE 2. Perioperative/postoperative events (for propensity score matched sets data)

\begin{tabular}{|c|c|c|c|c|c|}
\hline \multirow[b]{2}{*}{ Variable } & \multicolumn{2}{|c|}{$\begin{array}{c}\text { Obese } \\
(\mathbf{n}=\mathbf{1 2 3 8})\end{array}$} & \multicolumn{2}{|c|}{$\begin{array}{l}\text { Nonobese } \\
(\mathrm{n}=6179)\end{array}$} & \multirow{2}{*}{$\begin{array}{c}P \\
\text { value }\end{array}$} \\
\hline & Count & $\%$ & Count & $\%$ & \\
\hline \multicolumn{6}{|l|}{ Disposition } \\
\hline Routine & 839 & 67.8 & 4396 & 71.2 & .003 \\
\hline ICF & 383 & 31.0 & 1650 & 26.7 & \\
\hline Died & 15 & 1.2 & 124 & 2.0 & \\
\hline Prolonged LOS* & 79 & 6.4 & 529 & 8.6 & .011 \\
\hline \multicolumn{6}{|l|}{ Postoperative morbidity } \\
\hline Acute MI & 1 & 0.1 & 13 & 0.2 & .338 \\
\hline $\begin{array}{l}\text { Atrial fibrillation/ } \\
\text { flutter }\end{array}$ & 189 & 15.3 & 906 & 14.7 & .585 \\
\hline $\begin{array}{l}\text { Other cardiac } \\
\text { complications }\end{array}$ & 56 & 4.5 & 285 & 4.6 & .891 \\
\hline Pulmonary embolus & 4 & 0.3 & 17 & 0.3 & .772 \\
\hline $\begin{array}{l}\text { Pulmonary } \\
\text { insufficiency }\end{array}$ & 118 & 9.5 & 476 & 7.7 & .031 \\
\hline Pneumonia & 244 & 19.7 & 1034 & 16.7 & .011 \\
\hline $\begin{array}{c}\text { Other respiratory } \\
\text { complications }\end{array}$ & 79 & 6.4 & 331 & 5.4 & .150 \\
\hline Stroke & 1 & 0.1 & 17 & 0.3 & .205 \\
\hline $\begin{array}{l}\text { Other nervous system } \\
\text { complications }\end{array}$ & 1 & 0.1 & 4 & 0.1 & .843 \\
\hline ARF & 26 & 2.1 & 125 & 2.0 & .861 \\
\hline UTI & 37 & 3.0 & 133 & 2.2 & .073 \\
\hline $\begin{array}{l}\text { Other renal } \\
\text { complications }\end{array}$ & 11 & 0.9 & 61 & 1.0 & .747 \\
\hline GI complications & 24 & 1.9 & 112 & 1.8 & .763 \\
\hline $\begin{array}{l}\text { Venous DVT lower } \\
\text { extremity }\end{array}$ & 1 & 0.1 & 10 & 0.2 & .499 \\
\hline $\begin{array}{l}\text { Other vascular } \\
\text { complications }\end{array}$ & 1 & 0.1 & 6 & 0.1 & .864 \\
\hline $\begin{array}{l}\text { Infectious } \\
\text { complications }\end{array}$ & 5 & 0.4 & 29 & 0.5 & .756 \\
\hline
\end{tabular}

$\overline{I C F}$, Institutional care facility; $L O S$, length of stay; $M I$, myocardial infarction; $A R F$, acute renal failure; UTI, urinary tract infection; GI, gastrointestinal; DVT, deep venous thrombosis. *PLOS if more than 14 days.

$P=.03)$ and preoperative coronary artery disease (OR, 1.98; 95\% CI, 1.22-3.24; $P<.01$ ). Only the postoperative morbidities pulmonary insufficiency and renal complications were significantly associated with increased odds of death.

Likewise, we examined the likelihood of PLOS between obese and nonobese groups among patients with routine disposition. The models were adjusted for important relevant confounders as described previously. The results are summarized in Table 5. In the cohort of patients with routine disposition, the odds of PLOS were not different between the groups of obese and nonobese patients (OR, 0.79; $95 \%$ CI, $0.50-1.22 ; P=.28$; Table 5 ). As expected, all postoperative morbidities were associated with increased odds of PLOS (all $P<.05$ ), except for other cardiac complications $(P=.43)$ and gastrointestinal complications $(P=.50)$. Among preoperative comorbidities, chronic 
TABLE 3. Predictors of discharge to an institutional care facility (for propensity score matched sets data)

\begin{tabular}{|c|c|c|c|}
\hline Variable & OR & 95\% CI & $P$ value \\
\hline Obese & 1.214 & $1.033-1.426$ & .018 \\
\hline Age & 1.048 & $1.039-1.057$ & $<.001$ \\
\hline Sex & 1.515 & $1.328-1.729$ & $<.001$ \\
\hline Race, black & 1.281 & $1.019-1.61$ & .034 \\
\hline Race, other & 0.815 & $0.630-1.055$ & .121 \\
\hline Hospital type, urban nonteaching & 0.973 & $0.730-1.297$ & .853 \\
\hline Hospital type, urban teaching & 0.850 & $0.640-1.128$ & .260 \\
\hline $\begin{array}{l}\text { Primary expected payer, private/HMO, } \\
\text { Medicare/Medicaid }\end{array}$ & 0.707 & $0.600-0.833$ & $<.001$ \\
\hline Primary expected payer, self & 0.451 & $0.251-0.811$ & .008 \\
\hline Primary expected payer, other & 0.668 & $0.402-1.110$ & .120 \\
\hline \multicolumn{4}{|l|}{ Preoperative morbidity } \\
\hline Hypertension & 0.969 & $0.845-1.112$ & .656 \\
\hline Coronary artery disease & 1.134 & $0.967-1.329$ & .122 \\
\hline $\mathrm{CHF}$ & 1.698 & $1.267-2.275$ & $<.001$ \\
\hline PVD unspecified & 1.051 & $0.694-1.59$ & .815 \\
\hline COPD & 1.221 & $1.075-1.387$ & .002 \\
\hline GERD & 1.051 & $0.895-1.233$ & .547 \\
\hline Other (diabetes, CKD) & 1.573 & $1.136-2.178$ & .006 \\
\hline \multicolumn{4}{|l|}{ Postoperative morbidity } \\
\hline Atrial fibrillation/flutter & 1.292 & $1.074-1.554$ & .007 \\
\hline Other cardiac complications* & 1.351 & $1.000-1.825$ & .050 \\
\hline Pulmonary insufficiency & 1.804 & $1.421-2.289$ & $<.001$ \\
\hline Pneumonia & 1.151 & $0.958-1.383$ & .132 \\
\hline Other respiratory complications $\dagger$ & 1.451 & $1.088-1.934$ & .011 \\
\hline $\begin{array}{r}\text { Renal complications } \\
\text { (other, UTI, ARF) }\end{array}$ & 1.885 & $1.421-2.501$ & $<.001$ \\
\hline GI complications & 1.453 & $0.930-2.268$ & .101 \\
\hline Other complications $\ddagger$ & 2.191 & $1.180-4.069$ & .013 \\
\hline \multicolumn{4}{|c|}{$\begin{array}{l}O R \text {, Odds ratio; } C I \text {, confidence interval; } H M O \text {, Health Maintenance Organization; } \\
C H F \text {, congestive heart failure; } P V D \text {, peripheral vascular disease; } C O P D \text {, chronic } \\
\text { obstructive pulmonary disease; GERD, gastroesophageal reflux disease; } C K D \text {, } \\
\text { chronic kidney disease; } U T I \text {, urinary tract infection; } A R F \text {, acute renal failure; } G I \text {, gas- } \\
\text { trointestinal. *Includes } 56 \text { acute myocardial infarctions. } \dagger \text { Includes } 123 \text { pulmonary } \\
\text { emboli. } \ddagger \text { Includes stroke, nervous system other, vascular complication other, deep } \\
\text { venous thrombosis lower extremity, and infectious complication. }\end{array}$} \\
\hline
\end{tabular}

obstructive pulmonary disease was associated with increased likelihood of PLOS $(P<.05)$.

\section{DISCUSSION}

Obesity intuitively would seem to be an independent risk factor for major surgery. Hypoventilation syndromes, cardiac arrhythmias, wound-healing complications, and proinflammatory states are common in the obese patient populations, ${ }^{3,4}$ but other metabolic abnormalities also are prevalent, such as deficiencies in protein synthesis efficiency and elevated muscle catabolism in response to trauma. ${ }^{11}$ In the trauma population, obese patients exhibit a higher risk of mortality and morbidity compared with nonobese patients. ${ }^{12,13}$ Although most nonbariatric general surgical studies examining the impact of obesity on perioperative outcome demonstrate an increased risk for morbidity, including atrial fibrillation, surgical site infection, and embolism, there are few data to support an increase in mortality. ${ }^{5,6}$
TABLE 4. Predictors of mortality (for propensity score matched sets data)

\begin{tabular}{|c|c|c|c|}
\hline Variable & OR & 95\% CI & $P$ value \\
\hline Obese & 0.627 & $0.333-1.180$ & .148 \\
\hline Age & 1.034 & $1.004-1.066$ & .028 \\
\hline Sex & 1.041 & $0.660-1.640$ & .864 \\
\hline Race, black & 1.394 & $0.656-2.963$ & .388 \\
\hline Race, other & 1.574 & $0.734-3.372$ & .244 \\
\hline Hospital type, urban nonteaching & 0.408 & $0.183-0.910$ & .028 \\
\hline Hospital type, urban teaching & 0.296 & $0.131-0.670$ & .004 \\
\hline $\begin{array}{l}\text { Primary expected payer, private/ } \\
\text { HMO, Medicare/Medicaid }\end{array}$ & 0.811 & $0.426-1.546$ & .525 \\
\hline Primary expected payer, self & 1.443 & $0.349-5.960$ & 612 \\
\hline Primary expected payer, other & 1.516 & $0.338-6.800$ & .587 \\
\hline \multicolumn{4}{|l|}{ Preoperative morbidity } \\
\hline Hypertension & 0.784 & $0.495-1.241$ & .299 \\
\hline Coronary artery disease & 1.984 & $1.215-3.240$ & .006 \\
\hline $\mathrm{CHF}$ & 1.958 & $1.063-3.607$ & .031 \\
\hline PVD unspecified & 1.913 & $0.634-5.770$ & .250 \\
\hline COPD & 0.953 & $0.609-1.492$ & .834 \\
\hline GERD & 0.813 & $0.404-1.637$ & .562 \\
\hline Other (diabetes, CKD) & 0.902 & $0.335-2.433$ & .839 \\
\hline \multicolumn{4}{|l|}{ Postoperative morbidity } \\
\hline Atrial fibrillation/flutter & 1.002 & $0.580-1.732$ & .993 \\
\hline Other cardiac complications* & 1.843 & $0.865-3.929$ & .113 \\
\hline Pulmonary insufficiency & 13.087 & $7.993-21.427$ & $<.001$ \\
\hline Pneumonia & 1.032 & $0.608-1.753$ & .906 \\
\hline $\begin{array}{r}\text { Other respiratory } \\
\text { complications } \dagger\end{array}$ & 1.632 & 0.830-3.209 & .156 \\
\hline $\begin{array}{r}\text { Renal complications } \\
\text { (other, UTI, ARF) }\end{array}$ & 3.688 & $2.110-6.445$ & $<.001$ \\
\hline GI complications & 0.344 & $0.072-1.64$ & .181 \\
\hline Other complications $\ddagger$ & 2.838 & $0.959-8.404$ & .060 \\
\hline
\end{tabular}

$O R$, Odds ratio; $C I$, confidence interval; $H M O$, Health Maintenance Organization; $C H F$, congestive heart failure; $P V D$, peripheral vascular disease; $C O P D$, chronic obstructive pulmonary disease; $G E R D$, gastroesophageal reflux disease; $C K D$, chronic kidney disease; $U T I$, urinary tract infection; $A R F$, acute renal failure; $G I$, gastrointestinal. *Includes 56 acute myocardial infarctions. †Includes 123 pulmonary emboli. $\ddagger$ Includes stroke, nervous system other, vascular complication other, deep venous thrombosis lower extremity, and infectious complication.

The cardiothoracic surgical literature demonstrates mixed results. Although there are few data to support obesity as an independent risk factor for perioperative mortality, Zacharias and colleagues ${ }^{14}$ demonstrated an increased risk of atrial fibrillation in obese patients undergoing coronary artery bypass grafting. Kuduvalli et $\mathrm{al}^{15}$ found an increased risk of perioperative arrhythmia, sternal wound infections, and longer hospital stay in obese patients undergoing coronary artery bypass grafting. In regard to general thoracic surgery, Scipione et $\mathrm{al}^{8}$ retrospectively reviewed more than 2000 patients undergoing transhiatal esophagectomy, of whom 133 were profoundly obese (BMI $\geq 35$ $\mathrm{kg} / \mathrm{m}^{2}$ ), and found no difference in mortality, morbidity (except recurrent laryngeal nerve injury, which was higher in the obese group), hospital length of stay, or intensive care unit stay. For pulmonary resection, Smith et $\mathrm{al}^{7}$ found no difference in perioperative mortality or morbidity in 127 
TABLE 5. Predictors of prolonged length of stay (for propensity score matched sets data)

\begin{tabular}{|c|c|c|c|}
\hline Variable & OR & $\mathbf{9 5} \% \mathrm{CI}$ & $P$ value \\
\hline Obese & 0.787 & $0.509-1.218$ & .283 \\
\hline Age & 0.974 & $0.957-0.992$ & .004 \\
\hline Sex & 0.891 & $0.649-1.222$ & .473 \\
\hline Race, black & 1.393 & $0.848-2.290$ & .191 \\
\hline Race, other & 0.593 & $0.300-1.170$ & .132 \\
\hline Hospital type, urban nonteaching & 0.973 & $0.483-1.962$ & .940 \\
\hline Hospital type, urban teaching & 0.799 & $0.399-1.601$ & .527 \\
\hline $\begin{array}{c}\text { Primary expected payer, private/ } \\
\text { HMO, Medicare/Medicaid }\end{array}$ & 0.586 & $0.407-0.844$ & .004 \\
\hline Primary expected payer, self & 0.415 & $0.123-1.405$ & .158 \\
\hline Primary expected payer, other & 0.518 & $0.155-1.732$ & .286 \\
\hline \multicolumn{4}{|l|}{ Preoperative morbidity } \\
\hline Hypertension & 0.670 & $0.487-0.921$ & .014 \\
\hline Coronary artery disease & 0.731 & $0.471-1.133$ & .161 \\
\hline Heart failure & 1.037 & $0.475-2.264$ & .927 \\
\hline PVD unspecified & 0.970 & $0.332-2.837$ & .956 \\
\hline COPD & 1.374 & $1.003-1.881$ & .048 \\
\hline GERD & 0.613 & $0.376-1.001$ & .050 \\
\hline Other (diabetes, CKD) & 1.413 & $0.625-3.193$ & .407 \\
\hline \multicolumn{4}{|l|}{ Postoperative morbidity } \\
\hline Atrial fibrillation/flutter & 1.591 & $1.014-2.497$ & .044 \\
\hline Other cardiac complications* & 1.320 & $0.663-2.626$ & .429 \\
\hline Pulmonary insufficiency & 3.701 & $2.375-5.766$ & $<.001$ \\
\hline Pneumonia & 1.815 & $1.235-2.666$ & .002 \\
\hline $\begin{array}{r}\text { Other respiratory } \\
\text { complications } \dagger\end{array}$ & 1.823 & $1.043-3.187$ & .035 \\
\hline $\begin{array}{r}\text { Renal complications } \\
\text { (other, UTI, ARF) }\end{array}$ & 1.978 & $1.055-3.710$ & .034 \\
\hline GI complications & 1.401 & $0.526-3.726$ & .500 \\
\hline Other complications $\ddagger$ & 10.007 & $3.779-26.499$ & $<.001$ \\
\hline
\end{tabular}

$O R$, Odds ratio; $C I$, confidence interval; $H M O$, Health Maintenance Organization; $P V D$, peripheral vascular disease; $C O P D$, chronic obstructive pulmonary disease; $G E R D$, gastroesophageal reflux disease; $C K D$, chronic kidney disease; $U T I$, urinary tract infection; $A R F$, acute renal failure; $G I$, gastrointestinal. *Includes 56 acute myocardial infarctions. †Includes 123 pulmonary emboli. †Includes stroke, nervous system other, vascular complication other, deep venous thrombosis lower extremity, and infectious complication.

obese patients compared with 372 nonobese patients. By reviewing 154 patients undergoing pneumonectomy, Petrella et $\mathrm{al}^{16}$ found that obese patients (defined as BMI $\geq 25 \mathrm{~kg}$ / $\mathrm{m}^{2}, \mathrm{n}=93$ ) have a 5 -fold increase in respiratory complications. ${ }^{15}$

Our data, a national retrospective review of perioperative outcomes of 1238 obese and 31,983 nonobese patients undergoing lobectomy for cancer, found similar results to those of previous smaller single institution studies. We found that despite having fewer preoperative comorbidities and a higher predilection toward having their surgery performed at academic teaching hospitals, nonobese patients demonstrated higher perioperative mortality than obese patients and a higher incidence of PLOS. We found no difference in perioperative morbidity, including wound infections and deep venous thrombosis, but obese patients exhibited a higher incidence of respiratory complications compared with matched nonobese patients. Contrary to the adult cardiac surgery literature, ${ }^{14,15}$ obese patients did not have an increase incidence of atrial fibrillation/flutter compared with nonobese patients. In our study, obese patients demonstrated a higher propensity to be discharged to an ICF compared with nonobese patients. Obesity was in fact an independent risk factor for discharge to an ICF. This may explain the decreased incidence of PLOS in the obese population.

There are limitations to our analysis. The results of this study would suggest an obesity protection paradox; however, the obese patients may have been a highly selected group. HCUP-NIS does not provide cancer stage information or pulmonary function results, such as diffusion capacity for the lungs for carbon monoxide or forced expiratory volume in 1 second. There may have been stage inequities between groups, and the obese patients may have been selected for optimal lung function above recommended guidelines. Also, during the study period, there was no ICD-9-CM code for video-assisted thoracoscopic lobectomy used by the database; therefore it would be impossible to determine whether the obese cohort had a higher incidence of video-assisted thoracoscopic lobectomy, which has been shown to result in diminished perioperative complications. ${ }^{17,18}$ Obesity is defined as BMI $30 \mathrm{~kg} / \mathrm{m}^{2}$ or greater; however, other reports suggest that BMI may not be the most accurate measurement of obesity-related perioperative risk. Computed tomography quantitative measurement of visceral adiposity may identify at-risk populations independently of BMI. ${ }^{19,20}$

\section{CONCLUSIONS}

Despite having more preoperative comorbidities and a higher predilection to undergoing surgery at nonteaching hospitals compared with nonobese patients, outside of pulmonary complications, obese patients do not have an increased risk for postoperative morbidity, mortality, or PLOS after lobectomy for cancer. Obese patients undergoing lobectomy for cancer do have an independent increased odds of being discharged to an ICF rather than to home. Obesity should not be considered a prohibitive surgical risk factor for pulmonary resection. Therefore, obese patients should undergo surgical standard of care therapy for appropriately stage-specific lung cancer. However, given the independent increased risk of postoperative pulmonary insufficiency and pneumonia in obese patients, attention should be given to aggressive prophylactic respiratory therapy in the perioperative period.

\section{References}

1. Flegal KM, Carroll MD, Ogden CL, Johnson CL. Prevalence and trends in obesity among US adults, 1999-2000. JAMA. 2002;288:1723-7.

2. Flegal KM, Carroll MD, Kit BK, Ogden CL. Prevalence of obesity and trends in the distribution of body mass index among US adults, 1999-2010. JAMA. 2012; 307:491-7.

3. Flancbaum L, Choban PS. Surgical implications of obesity. Annu Rev Med. 1998; 49:215-34. 
4. Despres J-P, Lemieux I. Abdominal obesity and metabolic syndrome. Nature. 2006;444:882-7.

5. Mullen JT, Davenport DL, Hutter MM, Hosokawa PW, Henderson WG, Khuri SF, et al. Impact of body mass index on perioperative outcomes in patients undergoing major intra-abdominal cancer surgery. Ann Surg Oncol. 2008;15:2164-72.

6. Merkow RP, Bilimoria KY, McCarter MD, Bentrem DJ. Effect of body mass index on short-term outcomes after colectomy for cancer. JAm Coll Surg. 2009;208:53-61.

7. Smith PW, Wang H, Gazoni LM, Shen KR, Daniel T, Jones DR. Obesity does not increase complications after anatomic resection for non-small cell lung cancer. Ann Thorac Surg. 2007;84:1098-106.

8. Scipione CN, Chang AC, Pickens A, Lau CL, Orringer MB. Transhiatal esophagectomy in the profoundly obese: implications and experience. Ann Thorac Surg. 2007;84:376-83.

9. Healthcare Cost and Utilization Project. Available at: http://www.hcup-us.ahrq. gov. Accessed March 17, 2012.

10. Rosenbaum PR, Rubin DB. The central role of propensity score in observational studies for causal effects. Biometrika. 1983;70:41-55.

11. D'Agostino RB Jr. Propensity score methods for bias reduction in the comparison of a treatment to non-randomized control group. Stat Med. 1998;17:2265-81.

12. Jeevanandam M, Young DH, Schiller WR. Obesity and the metabolic response to severe multiple trauma in man. J Clin Invest. 1991;87:262-9.

13. Brown CV, Neville AL, Rhee P, Salim A, Velmahos GC, Demetriades D. The impact of obesity on the outcomes of 1,153 critically injured blunt trauma patients. J Trauma. 2005;59:1048-51.

14. Zacharias A, Schwann TA, Riordan CJ, Durham SJ, Shah AS, Habib RH. Obesity and risk of new-onset atrial fibrillation after cardiac surgery. Circulation. 2005; 112:3247-55.

15. Kuduvalli M, Grayson AD, Oo AY, Fabri BM, Rashid A. The effect of obesity on mid-term survival following coronary artery bypass surgery. Eur J Cardiothorac Surg. 2002;22:787-93

16. Petrella F, Radice D, Borri A, Galetta D, Gasparri R, Solli P, et al. The impact of preoperative body mass index on respiratory complications after pneumonectomy for non-small-cell lung cancer. Results from a series of 154 consecutive standard pneumonectomies. Eur J Cardiothorac Surg. 2011;39:738-44.

17. Flores RM, Park BJ, Dycoco J, Aronova A, Hirth Y, Rizk NP, et al. Lobectomy by video-assisted thoracic surgery (VATS) versus thoracotomy for lung cancer. J Thorac Cardiovasc Surg. 2009;138:11-8.

18. Park HS, Detterbeck FC, Boffa DJ, Kim AW. Impact of hospital volume of thoracoscopic lobectomy on primary lung cancer outcomes. Ann Thorac Surg. 2012; 93:372-9.

19. Kobayashi J, Tadokoro N, Watanabe M, Shinomiya M. A novel method of measuring intra-abdominal fat volume using helical computed tomography. Int J Obes Relat Metab Disord. 2002;26:398-402.

20. Hu FB. Obesity and mortality: watch your waist, not just your weight. Arch Intern Med. 2007;167:875-6.

APPENDIX 1. International Classification of Diseases, 9th edition, Clinical Modification codes

Preoperative Morbidity

25001 Diabetes mellitus

25002 Diabetes mellitus

25003 Diabetes mellitus

4010 Hypertension

4011 Hypertension

4019 Hypertension

41400 Coronary artery disease

41401 Coronary artery disease

4280 Heart failure

4289 Heart failure

4439 Peripheral vascular disease, unspecified

496 COPD (COPD/emphysema)

4928 Emphysema (COPD/emphysema)

53081 Esophageal reflux

5851 Chronic kidney disease

5852 Chronic kidney disease

\section{APPENDIX 1. Continued}

5853 Chronic kidney disease

5854 Chronic kidney disease

5855 Chronic kidney disease

5856 Chronic kidney disease

5859 Chronic kidney disease

Postoperative Morbidity

Cardiac complications

9971 Cardiac complications

4100 Acute MI

4101 Acute MI

4102 Acute MI

4103 Acute MI

4104 Acute MI

4105 Acute MI

4106 Acute MI

4107 Acute MI

4108 Acute MI

4109 Acute MI

42731 Atrial fibrillation (Afib/flutter)

42732 Atrial flutter (Afib/flutter)

Respiratory Complications

9973 Respiratory complications (respiratory complications other)

99739 Other respiratory complications (other)

$41510 \mathrm{PE}$ and infarction (pulmonary embolus)

41511 Iatrogenic PE and infarction (pulmonary embolus)

51881 Acute respiratory failure (pulmonary insufficiency)

51882 Other pulmonary insufficiency (pulmonary insufficiency)

51884 Acute on chronic respiratory failure (pulmonary insufficiency)

5184 Acute edema of lung, unspecified (pulmonary insufficiency)

5185 Pulmonary insufficiency after trauma and surgery (pulmonary

insufficiency)

485 Pneumonia

486 Pneumonia

4820 Pneumonia

4821 Pneumonia

4822 Pneumonia

48230 Pneumonia

48231 Pneumonia

48232 Pneumonia

48239 Pneumonia

48240 Pneumonia

48241 Pneumonia

48242 Pneumonia

48249 Pneumonia

48281 Pneumonia

48282 Pneumonia

48283 Pneumonia

48289 Pneumonia

4829 Pneumonia

5180 Atelectasis

Nervous System Complications

99700 Nervous system complications unspecified (nervous system other)

99701 Central nervous system complication (other)

99702 Iatrogenic cerebrovascular infarction or hemorrhage (stroke) 99709 Other nervous system complications (other)

(Continued) 


\section{APPENDIX 1. Continued}

Renal Complications

9975 Urinary complications (renal complications other)

5845 ARF

5846 ARF

5847 ARF

5849 ARF

5990 UTI

GI Complications

9974 Digestive system complications (GI complications other)

5601 Paralytic ileus

Vascular Complications

9972 Peripheral vascular complications (vascular complications other)

45340 Venous DVT lower extremity

45341 Venous DVT lower extremity

45342 Venous DVT lower extremity

Other Infectious Complications

99851 Infected postoperative seroma

99859 Other postoperative infection

Wound

99883 Nonhealing surgical wound

Other

9989 Complications affecting other specified body systems, not elsewhere classified

COPD, Chronic obstructive pulmonary disease; $M I$, myocardial infarction; Afib, atrial fibrillation; $P E$, pulmonary embolism; $A R F$, acute renal failure; $U T I$, urinary tract infection; $G I$, gastrointestinal; $D V T$, deep vein thrombosis. 